\title{
Effect of Icosapent Ethyl (Eicosapentaenoic Acid Ethyl Ester) on Omeprazole Plasma Pharmacokinetics in Healthy Adults
}

\author{
Rene A. Braeckman • William G. Stirtan • \\ Paresh N. Soni
}

Published online: 28 June 2014

(c) The Author(s) 2014. This article is published with open access at Springerlink.com

\begin{abstract}
Background Icosapent ethyl (IPE) is a high-purity prescription form of eicosapentaenoic acid ethyl ester approved by the US Food and Drug Administration as an adjunct to diet to reduce triglyceride levels in adult patients with severe hypertriglyceridemia. Patients with high serum triglycerides may be taking concurrent medications for associated conditions such as obesity and/or diabetes mellitus.

Objective To evaluate the effect of IPE on the plasma pharmacokinetics (PK) of omeprazole, a commonly used proton pump inhibitor and a substrate of cytochrome $\mathrm{P} 450$ (CYP) 2C19.

Study design Omeprazole (40 mg/day for 7 days) was administered orally without and with $4 \mathrm{~g} /$ day IPE at steady state. The primary PK endpoint was area under the concentration-time curve from time 0 to $24 \mathrm{~h}$ ( $\mathrm{AUC}_{0-24}$ ); secondary endpoints included maximum observed plasma concentration $\left(C_{\max }\right)$. Safety was monitored in all subjects who received one or more dose(s) of the study drug.

Participants Thirty healthy adult subjects were enrolled and 28 completed the study.

Results IPE $4 \mathrm{~g} /$ day at steady state did not significantly change the $\mathrm{AUC}_{0-24}$ or $C_{\text {max }}$ of omeprazole when coadministered at $40 \mathrm{mg} /$ day to steady state. The ratios of least squares geometric means (90\% confidence interval)
\end{abstract}

Drs. R. A. Braeckman and P. N. Soni are former employees of Amarin Pharma Inc., and were employed by Amarin Pharma Inc. during the planning, execution, and manuscript preparation of this study.

R. A. Braeckman · W. G. Stirtan ( ()$\cdot$ P. N. Soni

Amarin Pharma Inc., 1430 Route 206, Suite 200, Bedminster, NJ 07921, USA

e-mail: bill.stirtan@amarincorp.com for $\mathrm{AUC}_{0-24}$ and $C_{\max }$ (omeprazole with IPE vs. omeprazole alone) were 0.84 (76.0-91.9) and 1.01 (87.4-116.3), respectively. There were no clinically significant findings from laboratory tests, vital signs, or physical examinations. Conclusions At steady-state concentrations, IPE $4 \mathrm{~g} /$ day did not inhibit the $\mathrm{AUC}_{0-24}$ or $C_{\text {max }}$ of omeprazole $40 \mathrm{mg} /$ day, a CYP2C19 substrate. Co-administration of IPE with omeprazole was safe and well tolerated.

\section{Key Points}

Icosapent ethyl is a high-purity prescription form of eicosapentaenoic acid ethyl ester approved by the US Food and Drug Administration as an adjunct to diet to reduce triglyceride levels in adult patients with severe hypertriglyceridemia

Patients with high serum triglycerides may be taking concurrent medications including omeprazole, a widely used proton pump inhibitor and a competitive substrate of cytochrome P450 2C19

In this evaluation in healthy subjects, icosapent ethyl did not inhibit the plasma pharmacokinetics of omeprazole, and co-administration of the two drugs was safe and well tolerated

\section{Introduction}

Hypertriglyceridemia is common among adults in the USA, mainly owing to the prevalence of obesity and diabetes 
mellitus [1-3]. Individuals with elevated serum triglycerides (TG) often take multiple medications concomitantly for associated medical conditions [1]. Therefore, it is important for TG-lowering therapies to be well characterized with respect to possible drug-drug interactions to avoid any clinically significant effects when co-administered with other therapies.

Icosapent ethyl (IPE; Vascepa ${ }^{\circledR}$ [formerly AMR101]; Amarin Pharma Inc., Bedminster, NJ, USA) is a high-purity prescription form of eicosapentaenoic acid (EPA) ethyl ester approved by the US Food and Drug Administration (FDA) as an adjunct to diet to reduce TG levels in adult patients with severe $(\geq 5.65 \mathrm{mmol} / \mathrm{L})$ hypertriglyceridemia [4]. The safety and efficacy of IPE were established in the Multi-center, plAcebo-controlled, Randomized, doubleblINd, 12-week study with an open-label Extension (MARINE) and ANCHOR studies, which investigated the effects of IPE in patients with very high serum TG levels $(\geq 5.65 \mathrm{mmol} / \mathrm{L}$ and $\leq 22.6 \mathrm{mmol} / \mathrm{L})$ and in high-risk statintreated patients with high TG levels $(\geq 2.26$ and $<5.65 \mathrm{mmol} / \mathrm{L}$ ) despite having well-controlled low-density lipoprotein cholesterol (LDL-C) levels $(\geq 1.04$ and $<2.59 \mathrm{mmol} / \mathrm{L}$ ), respectively [5, 6]. In both studies, IPE at the approved dose of $4 \mathrm{~g}$ /day was found to significantly reduce serum TG levels and improve other lipid parameters without significantly increasing LDL-C levels [5, 6].

IPE is de-esterified during absorption, and peak plasma concentrations of EPA (active metabolite) are reached approximately $5 \mathrm{~h}$ following oral dosing [4]. Most of the EPA in the plasma is incorporated in phospholipids, TGs, and cholesteryl esters; $<1 \%$ of the total EPA is unesterified [4]. EPA is metabolized mainly by beta oxidation with cytochrome P450 (CYP)-mediated metabolism as a minor pathway of elimination [4]. No clinically significant pharmacokinetic (PK) drug-drug interactions have been observed with the CYP3A4, CYP2C8, and CYP2C9 substrates atorvastatin, rosiglitazone, and warfarin, respectively [4].

Omeprazole is a proton pump inhibitor that is widely used for the treatment of duodenal and gastric ulcers, gastroesophageal reflux disease (GERD), and erosive esophagitis [7, 8]. CYP2C19 is the principal enzyme involved in the metabolism of several proton pump inhibitors $[9,10]$. There are differences in the activity of CYP2C19 in different individuals, and omeprazole PK profiles may be influenced by CYP2C19 polymorphisms $[10,11]$. Omeprazole is a highly sensitive competitive substrate of CYP2C19, and is recommended in FDA guidance for use as a probe in drug-drug interaction studies in humans [12].

The objective of this study was to investigate the effect of IPE $4 \mathrm{~g}$ /day on the plasma PK of orally administered omeprazole $40 \mathrm{mg} / \mathrm{day}$ and the potential for a drug-drug interaction.

\section{Methods}

\subsection{Study Population}

Healthy non-smoking men and women $>18$ and $<55$ years of age were eligible if they had a body mass index (BMI) $>18$ and $\leq 35 \mathrm{~kg} / \mathrm{m}^{2}$ and were in good health as determined by medical history and medical examination. Women of childbearing potential were required to use an acceptable method of birth control, and were excluded if they were pregnant, nursing, or planning a pregnancy. All medications or dietary supplements with known or potential lipid-altering effects (including statins, niacin $>200 \mathrm{mg} /$ day, fibrates, ezetimibe, bile acid sequestrants, or medications, supplements or foods enriched with omega-3 fatty acids) were prohibited within 4 weeks prior to the first dose of study medication and until the end of the study. Subjects were required to discontinue consumption of fish or foods fortified with EPA and/or docosahexaenoic acid at least 1 week prior to the first dose. Use of any medication that could change plasma lipid fractions or affect EPA concentrations in these fractions was disallowed. Subjects who routinely used omeprazole or any other $\mathrm{H}+/ \mathrm{K}+$ ATPase inhibitors or antacids within 4 weeks prior to the beginning of the study were excluded.

\subsection{Study Design}

This single-center, open-label, phase I study used a crossover design to investigate possible drug-drug interactions between IPE at steady state and two different drugs metabolized by CYP2C class isozymes, omeprazole (CYP2C19) and rosiglitazone (CYP2C8). During a 28-day screening period, healthy adults were evaluated for eligibility and clinical laboratory testing was completed. All subjects then received the same treatment regimen, with omeprazole and rosiglitazone being administered sequentially and separated by 3 -day washout periods. Omeprazole was dosed on days 1-7, rosiglitazone on day 11, IPE on days 12-29, omeprazole on days 19-25, and rosiglitazone on day 29. Omeprazole PK parameters were determined on days 7 and 25 (without and with IPE, respectively). This report focuses only on the portion of the study that investigated omeprazole without and with IPE (days 1-7 and 12-25, respectively). The results of the rosiglitazone portion of the study will be reported separately. Because of the crossover design, the number of patients in the group that 
received omeprazole was the same as in the group that received omeprazole and IPE.

In healthy subjects, the elimination half-life of omeprazole is $0.5-1 \mathrm{~h}$ [8]. Omeprazole PK are nonlinear, with an increase in systemic availability after doses $>40 \mathrm{mg}$ or prolonged administration because of the effects of omeprazole on gastric $\mathrm{pH}$ and a saturable gastrointestinal firstpass effect $[8,13]$. The bioavailability of omeprazole increases slightly with repeated doses [8]. Therefore, to decrease variability and to maximize systemic exposure comparable to the clinical use of omeprazole, omeprazole $40 \mathrm{mg}$ was dosed for 7 days in the current study. PK sampling was conducted over a $24-\mathrm{h}$ period because of the short elimination half-life of omeprazole.

Omeprazole was provided as Prilosec ${ }^{\circledR} 40$-mg delayedrelease capsules (AstraZeneca Pharmaceuticals LP, Wilmington, DE, USA), which were dispensed in two separate bottles for dosing on days 1-7 and days 19-25. Omeprazole was taken once daily $1 \mathrm{~h}$ prior to the start of breakfast. IPE $4 \mathrm{~g} /$ day, the FDA-approved daily dose [4], was administered as two liquid-filled, 1-g gelatin capsules twice daily with or following the morning and evening meals on days 12-29. Treatments were self-administered when subjects were away from the study site, and administered by study personnel during scheduled visits. Compliance for at-home dosing was determined by study personnel by counting unused capsules and reconciling against subject diaries. Compliance was calculated as $100 \times$ the number of used capsules/total dosing days $\times 1$ for omeprazole (one capsule once daily) and $\times 4$ for IPE (two capsules twice daily).

The protocol was approved by an institutional review board (IntegReview Ethics Review Board, Austin, TX, USA) and the study was conducted between February 3, 2011 and March 21, 2011 at Frontage Clinical Services (a wholly owned subsidiary of Frontage Laboratories, Hackensack, NJ, USA). The study complied with the ethical principles of Good Clinical Practice and was conducted in accordance with the Declaration of Helsinki. All participants provided written informed consent prior to study entry.

\subsection{Pharmacokinetic Sampling and Bioanalytical Methods}

For determination of omeprazole plasma concentrations, blood samples $(6 \mathrm{~mL})$ were collected prior to the day 1 dose and on days 7 and 25 at time 0 (prior to dosing) and at $0.33,0.67,1,1.5,2,2.5,3,4,6,8,10,12,14$, and $24 \mathrm{~h}$ post-dose. Plasma was separated by centrifugation following collection of blood samples in prechilled glass tubes containing dipotassium ethylenediaminetetraacetic acid. Plasma concentrations of omeprazole were measured using a validated liquid chromatography with tandem mass spectrometry method by Frontage Laboratories, Inc. (Malvern, PA, USA). Omeprazole and omeprazole- $\mathrm{d}_{3}$ were extracted from human plasma by protein precipitation using acetonitrile and separated by reversed-phase highperformance liquid chromatography with a Gemini ${ }^{\circledR}$ C6Phenyl column (50x 2 mm, $5 \mu \mathrm{m}$; Phenomenex, Torrance, CA, USA) and Shimadzu HPLC pump and autosampler (Shimadzu, Kyoto, Japan), with a flow rate of $0.4 \mathrm{~mL} / \mathrm{min}$ at room temperature and an elution time of $1.4 \mathrm{~min}$. Mobile phase A was $2 \mathrm{mM}$ ammonium formate in $\mathrm{H}_{2} \mathrm{O}$ and mobile phase $\mathrm{B}$ was $2 \mathrm{~mm}$ ammonium formate in $\mathrm{MeOH}$. Omeprazole- $\mathrm{d}_{3}$ was used as the internal standard and the reference standard was omeprazole. Ions were monitored for omeprazole at $\mathrm{m} / \mathrm{z}, 346.3-198.1$ and for omeprazole- $\mathrm{d}_{3}$ at $\mathrm{m} / \mathrm{z}$ 349.1-198.1 in positive ionization mode using the API $4000^{\mathrm{TM}}$ mass spectrometer with TurboIonSpray electrospray ion source (AB Sciex, Framingham, MA, USA) at $575{ }^{\circ} \mathrm{C}$ and $5,500 \mathrm{~V}$ with $\mathrm{N}_{2}$. The dynamic range was $1-1,000 \mathrm{ng} / \mathrm{mL}$ with a lower limit of quantitation of $1 \mathrm{ng} /$ $\mathrm{mL}$. The assay accuracy (mean determined concentration/ nominal concentration) had a range of 93.0-99.8\% (intrarun) and 96.1-98.5\% (inter-run). The assay precision (coefficient of variation of the mean determined concentration) had a range of 0.6-3.7\% (intra-run) and 1.5-4.0\% (inter-run).

\subsection{Pharmacokinetic Evaluations and Statistical Methods}

WinNonlin version 5.0.1 or higher (Pharsight Corporation Inc., Mountain View, CA, USA) was used to derive PK parameters using standard non-compartmental analysis and actual sampling times. The primary PK endpoint for analysis of drug-drug interaction was the area under the plasma concentration-time curve from time 0 to $24 \mathrm{~h}$ $\left(\mathrm{AUC}_{0-24}\right)$ after multiple doses of omeprazole without (day 7) or with IPE at steady-state concentrations (day 25). Secondary PK endpoints included the maximum observed plasma concentration $\left(C_{\max }\right)$ and the time of occurrence of $C_{\max }\left(T_{\max }\right)$ for omeprazole. Additional endpoints included elimination half-life $\left(t_{1 / 2}\right)$ and apparent terminal elimination rate constant $\left(K_{\mathrm{el}}\right)$. Comparisons of the PK parameters for omeprazole without and with IPE included only subjects with values for the primary PK parameters available for omeprazole from both PK sampling days.

The intent-to-treat population included all subjects who signed the informed consent form and were included in the study. The PK population included all subjects who had available values for the primary omeprazole PK endpoint parameters from days 7 and 25 . The safety population included all subjects who received at least one dose of the 
study drug. A sample size of 30 subjects was planned to meet the aims of the study, allowing for up to six dropouts.

PK parameters were calculated by noncompartmental analysis using WinNonlin version 5.0.1 (Pharsight Corporation Inc., Mountain View, CA, USA). For each PK parameter, parametric and/or nonparametric descriptive statistics were calculated. Parametric statistics included mean, standard deviation (SD), geometric means, and percent coefficient of variation. Nonparametric statistics included median and range (minimum-maximum). Drugdrug interaction was based on the $\mathrm{AUC}_{0-24}$ of omeprazole. Analysis of variance models were used for analyzing AUC and $C_{\max }$ parameters based on natural log-transformed values. This included the effects for treatment (without or with IPE) as a random effect. The estimate of the ratio between the two treatments for these parameters and the corresponding $90 \%$ confidence intervals (CI) for the ratio were obtained by exponentiating the difference in logarithms, and were used to determine whether a drug-drug interaction of the two treatments (without or with IPE) occurred.

\subsection{Safety Assessments}

Safety evaluations consisted of monitoring adverse events (AEs), clinical laboratory measurements (chemistry, hematology, and urinalysis), vital signs (systolic and diastolic blood pressure, heart rate, respiratory rate, and oral body temperature), and physical examinations.

\section{Results}

\subsection{Study Participants}

Thirty healthy subjects were enrolled, all of whom were given at least one dose of the study drug and were included in the safety analysis. The mean age (SD) was 38.5 (10.2) years, and mean weight and BMI (SD) were 78.5 (13.9) kg and $27.5(3.6) \mathrm{kg} / \mathrm{m}^{2}$, respectively. Subjects were primarily white $(n=21 ; 70.0 \%)$ and black/African American $(n=7 ; 23.3 \%)$. Twenty-eight subjects completed the study and were included in the PK analyses. Two subjects discontinued the study; one was unable to comply with study requirements and one did not present to the clinic on day 7. Mean (SD) treatment compliance based on capsule counts was $100.3 \%$ (3.5) for the 30 subjects who received omeprazole and 98.4 (4.2) for the 28 who received IPE.

\subsection{Pharmacokinetics}

Omeprazole plasma concentration-time profiles were comparable whether the drug was administered alone or with IPE $4 \mathrm{~g} /$ day at steady-state concentrations (Fig. 1). Mean exposure $\left(\mathrm{AUC}_{0-24}\right)$ was slightly higher and mean $C_{\max }$ was slightly lower when omeprazole was administered without IPE than when administered with IPE (Table 1). Median $T_{\max }$ and mean $t_{1 / 2}$ were similar for the two treatments (Table 1). Results from statistical analyses of drug-drug interaction are summarized in Table 2.

\subsection{Safety}

There were no clinically significant findings from laboratory test results or following physical examination and vital sign assessments. All reported AEs were mild or moderate in severity and there were no discontinuations because of an AE.

\section{Discussion}

This drug-drug interaction study examined the effects of IPE on the PK of omeprazole. The ratio of least squares means for $\mathrm{AUC}_{0-24}$ and $C_{\max }$ (without or with IPE) and the resulting $90 \%$ CIs indicated that a regimen of IPE 4 g/day did not inhibit omeprazole PK. Administration of omeprazole alone or co-administered with IPE was well tolerated in healthy subjects. IPE is a prescription form of EPA ethyl ester and has been studied for potential CYP-mediated drug-drug interactions in healthy adults. In addition to the effects described herein for omeprazole (CYP2C19 substrate), the administration of IPE $4 \mathrm{~g} /$ day did not display a significant effect on the AUC or $C_{\max }$ of atorvastatin (CYP3A4 substrate), rosiglitazone (CYP2C8 substrate), or warfarin (CYP2C9 substrate) [4].

Patients with hypertriglyceridemia often have comorbidities including obesity, metabolic syndrome, and diabetes mellitus $[1,2]$. Obesity and metabolic syndrome are associated with erosive esophagitis [14-17], with obesity being a very strong independent risk factor for GERD

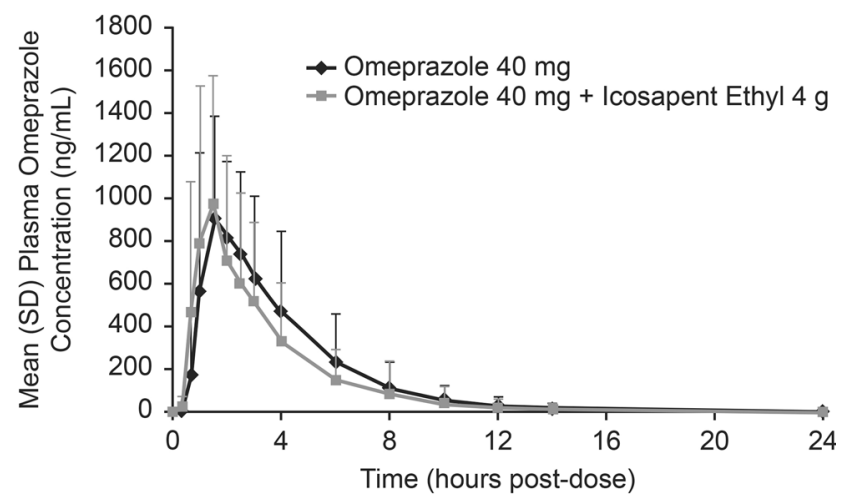

Fig. 1 Mean (SD) omeprazole $40 \mathrm{mg}$ /day plasma concentration-time curve when administered without or with icosapent ethyl $4 \mathrm{~g}$ /day (pharmacokinetic analysis population, $n=28$ ). $S D$ standard deviation 
Table 1 Pharmacokinetic parameters for omeprazole $40 \mathrm{mg} /$ day given without or with oral icosapent ethyl 4 g/day (pharmacokinetic analysis population, $n=28$ )

\begin{tabular}{lll}
\hline PK Parameter (unit) & Treatment & \\
\cline { 2 - 3 } & Omeprazole $40 \mathrm{mg}$ & Icosapent Ethyl 4 g + Omeprazole 40 mg \\
\hline $\mathrm{AUC}_{0-24}(\mathrm{ng} \cdot \mathrm{h} / \mathrm{mL})$ & $3,607(2,208)$ & $3,142(2,114)$ \\
$C_{\max }(\mathrm{ng} / \mathrm{mL})$ & $1,156(461)$ & $1,222(621)$ \\
$T_{\max }(\mathrm{h})$ & $1.5(1.0-6.0)$ & $1.5(0.7-8.0)$ \\
$t_{1 / 2}(\mathrm{~h})$ & $1.5(0.7)$ & $1.4(0.7)$ \\
$K_{\mathrm{el}}(\mathrm{L} / \mathrm{h})$ & $0.56(0.22)$ & $0.62(0.26)$ \\
\hline
\end{tabular}

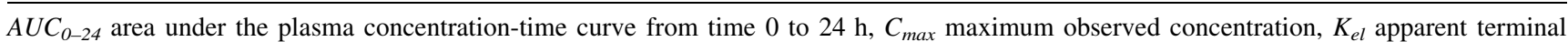
elimination rate constant, $P K$ pharmacokinetic, $t_{1 / 2}$ apparent terminal elimination half-life, $T_{\max }$ time of maximum observed concentration

${ }^{a}$ Mean (standard deviation) displayed for all PK parameters except $T_{\max }$, which is displayed as median (minimum-maximum)

Table 2 Statistical analysis of drug-drug interaction following omeprazole $40 \mathrm{mg} /$ day without or with oral icosapent ethyl $4 \mathrm{~g} / \mathrm{day}$ (pharmacokinetic analysis population, $n=28$ )

\begin{tabular}{llll}
\hline PK Parameter (unit) & Statistic $^{\mathrm{a}}$ & Treatment & Icosapent Ethyl $4 \mathrm{~g}+$ Omeprazole $40 \mathrm{mg}$ \\
\cline { 3 - 4 } & & Omeprazole $40 \mathrm{mg}$ & 2,484 \\
\hline $\mathrm{AUC}_{0-24}(\mathrm{ng} \cdot \mathrm{h} / \mathrm{mL})$ & LSGM & 2,973 & \\
& Ratio & 0.84 & 1,059 \\
$C_{\max }(\mathrm{ng} / \mathrm{mL})$ & $90 \% \mathrm{CI}$ & $75.99-91.87$ & \\
& LSGM & 1,051 & \\
& Ratio & 1.01 & \\
& $90 \% \mathrm{CI}$ & $87.36-116.3$ & \\
\hline
\end{tabular}

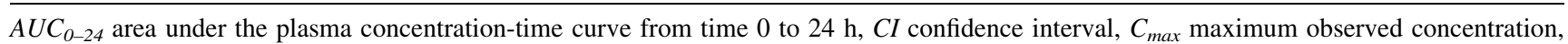
LSGM least squares geometric means, $P K$ pharmacokinetic

${ }^{\text {a }}$ LSGM derived from mixed models; LSGM ratios are provided for icosapent ethyl plus omeprazole/omeprazole alone

symptoms [14]. Consequently, many candidates for IPE TG-lowering therapy may be taking a concomitant medication for GERD or erosive esophagitis, such as omeprazole. Other proton pump inhibitors, including lansoprazole and esomeprazole, may also be involved in CYP2C19mediated metabolism [18]. Given its lack of effect on the PK of omeprazole, a CYP2C19 substrate, IPE may be a potential option for TG lowering in patients receiving proton pump inhibitor therapy.

A limitation of this study was that it did not investigate the potential effects of omeprazole on IPE through CYP2C19 inhibition or change in gastric $\mathrm{pH}$, although this is not expected. Another limitation was the relatively short study duration, given the potentially long duration of use of either one of the study drugs alone or when used concomitantly, although typically, drug-drug interaction studies are relatively short in duration.

\section{Conclusions}

At steady state, IPE $4 \mathrm{~g} /$ day did not inhibit the $\mathrm{AUC}_{0-24}$ and $C_{\max }$ of the CYP2C19 substrate omeprazole at $40 \mathrm{mg} /$ day. Coadministration of these two drugs was safe and well tolerated in this PK study of healthy adult subjects.

Acknowledgments This study was designed and sponsored by Amarin Pharma Inc., Bedminster, NJ, USA. Medical writing assistance was provided by Beth Daro-Kaftan, $\mathrm{PhD}$, of Peloton Advantage, LLC, Parsippany, NJ, USA, and funded by Amarin Pharma Inc.

Declaration of interest Dr. Stirtan is an employee and stock shareholder of Amarin Pharma Inc. Drs. Braeckman and Soni are former employees and current stock shareholders of Amarin Pharma Inc.

Open Access This article is distributed under the terms of the Creative Commons Attribution Noncommercial License which permits any noncommercial use, distribution, and reproduction in any medium, provided the original author(s) and the source are credited.

\section{References}

1. Ford ES, Li C, Zhao G, Pearson WS, Mokdad AH. Hypertriglyceridemia and its pharmacologic treatment among US adults. Arch Intern Med. 2009;169:572-8.

2. Third Report of the National Cholesterol Education Program (NCEP) Expert Panel on Detection, Evaluation, and Treatment of 
High Blood Cholesterol in Adults (Adult Treatment Panel III) final report. Circulation. 2002;106:3143-421.

3. Berglund L, Brunzell JD, Goldberg AC, Goldberg IJ, Sacks F, Murad MH, Stalenhoef AF. Evaluation and treatment of hypertriglyceridemia: an endocrine society clinical practice guideline. J Clin Endocrinol Metab. 2012;97:2969-89.

4. Vascepa [package insert]. Bedminster: Amarin Pharma Inc.; 2013.

5. Bays HE, Ballantyne CM, Kastelein JJ, Isaacsohn JL, Braeckman RA, Soni PN. Eicosapentaenoic acid ethyl ester (AMR101) therapy in patients with very high triglyceride levels (from the Multi-center, plAcebo-controlled, Randomized, double-blINd, 12-week study with an open-label Extension [MARINE] trial). Am J Cardiol. 2011;108:682-90.

6. Ballantyne CM, Bays HE, Kastelein JJ, Isaacsohn JL, Braeckman RA, Soni PN. Efficacy and safety of eicosapentaenoic acid ethyl ester (AMR101) therapy in statin-treated patients with persistent high triglycerides (from the ANCHOR study). Am J Cardiol. 2012;110:984-92.

7. Yacyshyn BR, Thomson AB. The clinical importance of proton pump inhibitor pharmacokinetics. Digestion. 2002;66:67-78.

8. Prilosec [package insert]. Wilmington: AstraZeneca Pharmaceuticals; 2012.

9. Abelo A, Andersson TB, Antonsson M, Naudot AK, Skanberg I, Weidolf L. Stereoselective metabolism of omeprazole by human cytochrome P450 enzymes. Drug Metab Dispos. 2000;28:966-72.

10. Furuta T, Shirai N, Sugimoto M, Nakamura A, Hishida A, Ishizaki T. Influence of CYP2C19 pharmacogenetic polymorphism on proton pump inhibitor-based therapies. Drug Metab Pharmacokinet. 2005;20:153-67.
11. Baldwin RM, Ohlsson S, Pedersen RS, Mwinyi J, IngelmanSundberg M, Eliasson E, Bertilsson L. Increased omeprazole metabolism in carriers of the CYP2C19*17 allele; a pharmacokinetic study in healthy volunteers. $\mathrm{Br} \mathrm{J}$ Clin Pharmacol. 2008;65:767-74.

12. Guidance for industry. Drug interaction studies: study design, data analysis, and implications for dosing and labeling. US Department of Health and Human Services; Food and Drug Administration, 2006. http://www.fda.gov/OHRMS/DOCKETS/ 98fr/06d-0344-gdl0001.pdf. Accessed 4 Feb 2014.

13. Rost KL, Roots I. Nonlinear kinetics after high-dose omeprazole caused by saturation of genetically variable CYP2C19. Hepatology. 1996;23:1491-7.

14. El-Serag HB, Graham DY, Satia JA, Rabeneck L. Obesity is an independent risk factor for GERD symptoms and erosive esophagitis. Am J Gastroenterol. 2005;100:1243-50.

15. Hampel H, Abraham NS, El-Serag HB. Meta-analysis: obesity and the risk for gastroesophageal reflux disease and its complications. Ann Intern Med. 2005;143:199-211.

16. Park JH, Park DI, Kim HJ, Cho YK, Sohn CI, Jeon WK, Kim BI. Metabolic syndrome is associated with erosive esophagitis. World J Gastroenterol. 2008;14:5442-7.

17. Kendall BJ, Macdonald GA, Hayward NK, Prins JB, O'Brien S, Whiteman DC. The risk of Barrett's esophagus associated with abdominal obesity in males and females. Int $\mathbf{J}$ Cancer. 2013;132:2192-9.

18. Zvyaga T, Chang SY, Chen C, Yang Z, Vuppugalla R, Hurley J, Thorndike D, Wagner A, Chimalakonda A, Rodrigues AD. Evaluation of six proton pump inhibitors as inhibitors of various human cytochromes P450: focus on cytochrome P450 $2 \mathrm{C} 19$. Drug Metab Dispos. 2012;40:1698-711. 